\title{
Inguinal Bladder Hernia with Bladder Carcinoma: An Unusual Manifestation
}

\author{
(1) Uygar Miçooğulları, (® Özgür Çakmak \\ University of Health Sciences Turkiye, Tepecik Training and Research Hospital, Clinic of Urology, Izmir, Turkiye
}

\begin{abstract}
It is extremely uncommon for bladder carcinoma to manifest in an inguinal hernia. Only 1-3\% of inguinal hernias involve the bladder, and very few of these hernias are associated with bladder carcinoma. Furthermore, the tumour is removed, and the hernia is repaired as part of the treatment. We present the case of an 89-year-old man who came to our clinic complaining of haematuria and was diagnosed with an inguinal bladder hernia filled with tumours.
\end{abstract}

Keywords: Inguinal hernia, bladder carcinoma, an unusual manifestation, case report, haematuria

\section{Introduction}

Inguinal hernias affect 3\%-8\% of the population (1). Inguinal bladder hernias are extremely rare, accounting for only 1\%-3\% of all inguinal hernias (1). The presence of carcinoma within the herniated portion of the bladder is an exceedingly rare entity that has only been anecdotally reported in the literature (2).

Here, we present a case of an 89-year-old man who had macroscopic haematuria and was later diagnosed with an inguinal bladder hernia filled with a tumour.

\section{Case Report}

An 89-year-old male patient arrived at our clinic complaining of haematuria. The results of the blood tests and kidney functions were both normal. Moreover, urine analysis and physical examination both supported macroscopic haematuria. The patient had no symptoms of the lower urinary tract, and digital rectal examination was normal. The urine culture was sterile, and the prostate-specific antigen level $(0.8 \mathrm{ng} / \mathrm{mL})$ was within the normal range. An abdominopelvic computed tomography with intravenous contrast substance revealed a tumour-filled inguinoscrotal bladder hernia (Figure 1). During the examination, it was discovered that the hernia could be manually reduced. Cystoscopy was performed, and papillary tumoural structures in the hernia sac were noted (Figure 2).

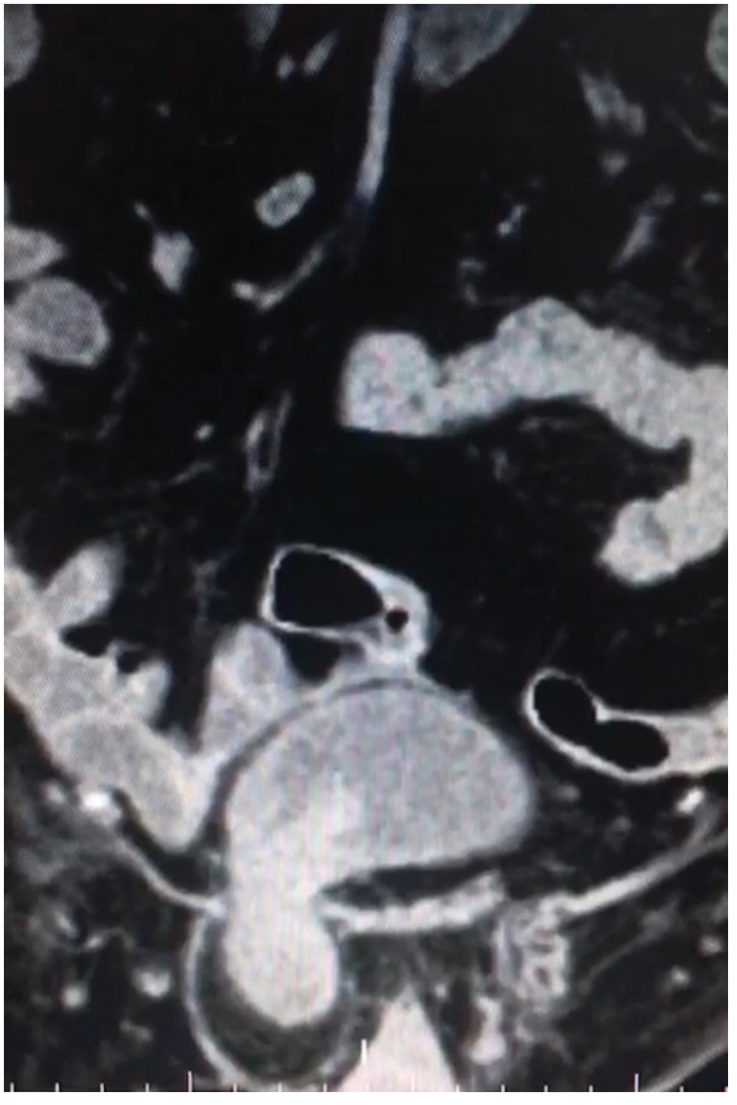

Figure 1. Abdominopelvic computed tomography showed a tumour-filled inguinoscrotal bladder hernia

Correspondence: Uygar Miçooğulları MD, University of Health Sciences Turkiye, Tepecik Training and Research Hospital, Clinic of Urology, İzmir, Turkiye Phone: +90 5435888989 E-mail: uygarmico@hotmail.com ORCID-ID: orcid.org/0000-0003-4729-6104

Received: 31.08 .20 Accepted: 28.10 .20

Cite this article as: Miçooğulları U, Çakmak Ö. Inguinal Bladder Hernia with Bladder Carcinoma: An Unusual Manifestation. J Urol Surg 2021;8(2):149-150. 


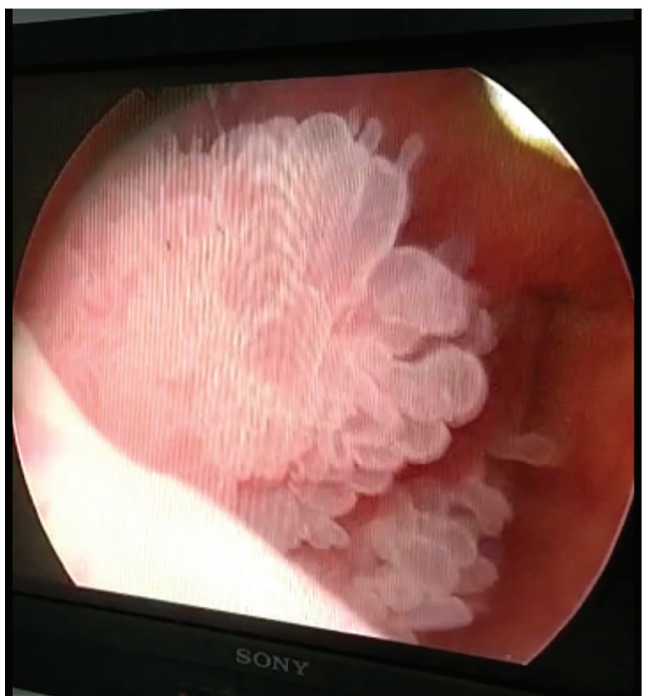

Figure 2. Hernia sac filled with papillary tumoural structures

Transurethral resection was used to remove the tumour. The pathology report revealed that the tumour was a low grade, non-invasive urothelial carcinoma. The patient was followed up in the urology department.

\section{Discussion}

Bladder herniation is a rare condition that accounts for only $1 \%-3 \%$ of all inguinal hernias (2). Bladder cancer in an inguinal hernia is an even more uncommon phenomenon (2). Levine first described scrotal cystocele as a hernia of the bladder to the scrotum in 1951 (3). Age, chronic bladder distension, loss of bladder tone, and factors that increase intraabdominal pressure, such as obesity, urinary tract obstruction, protrusion of perivesical fat, pelvic mass, or previous hernia surgery, are all known etiological factors (4).

Bladder herniation is usually asymptomatic and found incidentally, but it may present with urinary tract obstruction on rare occasions. It can also cause life-threatening complications such as bladder rupture, sepsis, cystolithiasis, hydronephrosis, vesicourethral reflux, kidney failure, and ischaemia-induced bladder infarction (5). During hernia surgery, the diagnosis is usually made. To avoid complications during surgical repair, it is critical to make an early diagnosis using adequate and detailed medical anamnesis and radiological imaging. According to Gomella et al. (6), approximately $38 \%$ of inguinal hernia surgeries resulted in unrecognised bladder injury. Moreover, postoperative complications such as gross haematuria, sepsis, or fistula formation occurred in these patients. In our case, the patient was admitted to the hospital with a complaint of macroscopic haematuria caused by a bladder tumour.

In the differential diagnosis, mesenteric cyst hernia, hydrocoele, bladder diverticulum, and spermatic cord cyst was considered
(7). The primary course of action is an endoscopic diagnosis, followed by tumour resection and bladder hernia repair (7). Alternatively, an inguinal hernia can also be repaired prior to tumour resection. In our case, since the hernia sac could be manually reduced, the resection of the bladder tumour was prioritised. Following a consultation with the general surgery clinic, it was determined that the patient's hernia did not require repair.

\section{Conclusion}

Inguinal bladder hernia is an uncommon condition, and the presence of tumours in an inguinal bladder hernia is even rarer (Medline/PubMed). The absence of findings specific to inguinal bladder tumours in the examination causes some difficulties during diagnosis. This could result in bladder injury during surgery. Therefore, radiological imaging is critical in the treatment of bladder tumours and the repair of inguinal hernias.

\section{Ethics}

Informed Consent: Patient's approval was taken.

Peer-review: Externally peer-reviewed.

\section{Authorship Contributions}

Surgical and Medical Practices: U.M., Ö.Ç., Concept: U.M., Ö.Ç., Design: U.M., Data Collection or Processing: U.M., Analysis or Interpretation: U.M., Ö.Ç., Literature Search: U.M., Writing: U.M.

Conflict of Interest: No conflict of interest was declared by the authors.

Financial Disclosure: The authors declared that this study received no financial support.

\section{References}

1. Zihni I, Duran A, Soysal V. A rare cause of inguinal hernia: scrotal cystocele. Ulus Cerrahi Derg 2014;32:137-139.

2. Ozman O, Aferin U, Çitgez S, Demirdag C, Talat C. Massive Inguinoscrotal Bladder Hernia and Bladder Tumor: A Very Rare Case. J Urol Surg 2018;5:202204.

3. LEVINE B. Scrotal cystocele. J Am Med Assoc 1951;147:1439-1441.

4. Moufid K, Touiti D, Mohamed L. Inguinal bladder hernia: four case analyses. Rev Urol 2013;15:32-36.

5. Bisharat M, O'Donnell ME, Thompson T, MacKenzie N, Kirkpatrick D, Spence RA, Lee J. Complications of inguinoscrotal bladder hernias: a case series. Hernia 2009;13:81-84.

6. Gomella LG, Spires SM, Burton JM, Ram MD, Flanigan RC. The surgical implications of herniation of the urinary bladder. Arch Surg 1985;120:964967.

7. Katsourakis A, Noussios G, Svoronos C, Alatsakis M, Chatzitheoklitos E. Direct inguinal hernia containing bladder carcinoma: A case report and review of the literature. Int J Surg Case Rep 2014;5:180-182. 